Oriani, R.A. Anomalous Heavy Atomic Masses Produced by Electrolysis. in The Seventh International Conference on Cold Fusion. 1998. Vancouver, Canada: ENECO, Inc., Salt Lake City, UT.

\title{
Anomalous Heavy Atomic Masses Produced by Electrolysis
}

\author{
R.A. Oriani \\ Department of Chemical Engineering and Materials Science \\ University of Minnesota \\ Minneapolis, MN 55455 \\ U.S.A.
}

\begin{abstract}
By applying to electrolysis cathodes a technique that produces essentially only oxides that are volatile at room temperature, spectroscopically determined masses between 222 and 351 are found that cannot be ascribed to known compounds. In particular the masses found between 231 and 240 AMU cannot be ascribed to random signals but do correspond to $\mathrm{CO}_{2}$ the carbon of which is a neutron-rich nuclide as predicted by a recent theory of polyneutron nuclear reactions.
\end{abstract}

\section{INTRODUCTION}

The announcement of Pons and Fleischmann (1) of the generation of thermal energy accompanying the electrolysis of heavy water upon palladium and their claim that nuclear fusion is the causative phenomenon produced a largely unbelieving and hostile reaction. This was caused by the strong contradiction between the claim of nuclear fusion occurring at ambient temperatures and the well established science of nuclear fusion, and also by the many negative results of the great majority of attempted replications of the Pons-Fleischmann experiments. Nevertheless, since that time a number of reliable, credible experiments have verified that thermal energy in excess of the energy supplied to the apparatus can indeed be generated by electrolysis, and the signature of a nuclear reaction such as helium generation, has been observed. (For a review see ref. 2). However, a continuing problem and impediment to the acceptance by the scientific community of the reality of the phenomenon is the irreproducibility of the experimental results. In the hands of most contemporary experimentalists in this field, the proportion of successful experiments producing excess energy is $10 \%$ or less, although there are now some who claim essentially $100 \%$ rate of success.

On the theoretical side there have been many attempts to develop mechanisms by which the Coulombic repulsion between approaching nuclei might be overcome or circumvented. Many of these theories contradict each other and are based on speculative ideas. The seeming impossibility of overcoming the Coulomb barrier at ambient temperatures has led some theorists to invoke neutral species as one of the reactants in nuclear reactions. Among these is Fisher, $(3,4)$ who espouses polyneutrons as the active agents in ambient-temperature nuclear reactions. The neutron clusters are supposed bound by a BCS-type of interaction; their multiplication and growth produce the excess thermal energy seen by some investigators and their decay by $\beta$ emission and fission produces the nuclides found after electrolysis by Miley et al. $(5,6)$ and by Mizuno et al. (7). Strange and iconoclastic as Fisher's theory appears, it has seemed worthwhile 
to consider it seriously because it is the only extant model that is compatible with the gaps in the distribution of nuclide masses observed after electrolysis both by Mizuno et al. and by Miley, et.al.

One of the predictions of the theory of Fisher is that heavy nuclides rich in neutrons should be generated, provided that certain conditions are satisfied. If such anomalous nuclides are indeed produced, their existence cannot be verified by any technique that depends on electronic transitions since the electronic characteristics of such putative nuclides are not known. The existence of anomalous masses may be revealed by secondary ion mass spectroscopy of mass resolution sufficiently high to distinguish from complexes of ordinary nuclides, but the chemical identity of the anomalous masses cannot be ascertained thereby. A chemical technique is necessary. In this paper is reported a chemical approach that attempts to verify the generation of neutron-rich carbon and nitrogen.

\section{EXPERIMENTAL}

Of the neutron-rich nuclides predicted by Fisher's theory (Figure 1) only carbon and nitrogen would produce oxides that are gases at room temperature. The technique adopted therefore is to heat in oxygen gas a metal cathode previously used in electrolytic deposition of deuterium or hydrogen. At a sufficiently high temperature the heavy carbon and nitrogen supposedly produced by the electrolysis should diffuse to the sample surface and there be oxidized to form $\mathrm{CO}_{2}$ and $\mathrm{NO}_{2}$. These gases can be collected in a portion of the apparatus maintained at liquid nitrogen temperature. Figure 2 is a schematic of the system employed. The sample, cut into small pieces is placed in a silica tube surrounded by chromel heating wire enclosed by thermal insulation. About 0.8 atm of oxygen is introduced and the contents are heated to between 1020 and $1050^{\circ} \mathrm{C}$ for 10 hours or longer. The heated zone produces continuous convection that takes the gaseous oxides to the neighborhood of the side-branching tube which is kept immersed in liquid nitrogen. Therefore a diffusive flux of the gaseous oxides is set up through the open stopcock towards the frozen condensate within the cold finger. After 10 to 12 hours the system is evacuated, leaving the condensed oxides of the putative heavy carbon and nitrogen, as well as ordinary $\mathrm{CO}_{2}, \mathrm{NO}_{2}$, and $\mathrm{H}_{2} \mathrm{O}$ or $\mathrm{D}_{2} \mathrm{O}$. The stopcock of the cold finger is then closed and the cold finger is removed from the apparatus. The same procedure is then applied to a sample of the same metal but which has not served as a cathode in electrolysis. The contents of the cold finger from this second run serve as the blank against which the results of the oxidation of the cathode are compared. The contents of the cold fingers now at room temperature are examined sequentially in a Finnigan MAT-95 high-resolution mass spectrometer. The ability to extract ordinary carbon from a metal by this procedure was confirmed by the use of a mild steel as the heated specimen. Carbon dioxide was identified by the mass spectrometer.

It is expected that masses produced by contaminants in the mass spectrometer, in the oxygen, in the metal and in the heating apparatus will appear both in the gases from the cathode sample and in those of the blank. However, sometimes lines appear in the spectrum of the blank that do not appear in the spectrum of the specimen. The reason for this is that gas flow from the collection vial into the ionization chamber of the mass spectrometer can desorb molecules residing in the instrument from previous use. Because the blank is analyzed first, these impurities appear in its spectrum and may not appear in the spectrum of the specimen. Therefore, only 
masses whose lines do not appear from the blank but do appear from the sample, as well as those which appear strongly from the sample but appear only insignificantly in the blank, are counted as significant.

\section{EXPERIMENTAL RESULTS AND DISCUSSION}

The described procedure has been applied to four metal cathodes and their corresponding blanks. The palladium sample designated 0-113 was shown (8) calorimetrically to have generated excess thermal power during electrolysis. It may be relevant to note that directly after the calorimetry this sample was cleaned in hydrochloric acid in a misguided attempt to clean the surface. The palladium samples designated by T-4 and T-7 were obtained from Prof. A. Takahashi, Osaka University. Both samples supported the generation of excess thermal power, as described in ref. (9). The nickel sample served as cathode for the electrolysis of $\mathrm{H}_{2} \mathrm{O}$ plus $\mathrm{D}_{2} \mathrm{O}$ in contrast to each of the Pd cathodes for which only $\mathrm{D}_{2} \mathrm{O}$ was used. The $\mathrm{Ni} / \mathrm{H}_{2} \mathrm{O}, \mathrm{D}_{2} \mathrm{O}$ experiment was patterned in part after the work of Patterson (10), who showed that excess thermal power can be developed with $\mathrm{Ni} / \mathrm{H}_{2} \mathrm{O}$ electrolysis. However, during our electrolysis, which lasted 16 days, no attempt was made to ascertain whether or not excess thermal power was developed.

Mass spectroscopic analysis of the blank (non-cathode metal sample) was carried out first, immediately followed by the analysis of the cathode sample. Multiple mass scans were made on each and the scans were averaged by the instrument in order to reduce the effect of instrumental noise. The averaged mass spectrum from the cathode was carefully compared with the averaged mass spectrum from the corresponding blank, and the individual mass scans were also examined for consistency in displaying a mass appearing from the cathode specimen but not appearing in the blank. The results of these examinations are shown in Table I. The mass values enclosed in parentheses are those for which a signal somewhat above noise level appears in the blank mass spectrum but for which the line for that mass in the cathode spectrum appears much stronger. Figure 3 shows a typical example. The mass values in Table I not in parentheses are those for which not trace at all appears in the corresponding blank, thereby effectively eliminating any possibility that these lines arise from contamination. Figure 3 shows a typical example of this category also.

To see if the mass values in Table 1 can be ascribed to known compounds we have searched the literature for compounds having molecular masses within 0.9 AMU from the mass values in Table I. These are collected in Table II, together with the mass values from Table I with which they may be related. However, candidate compounds must be stable at $1020^{\circ} \mathrm{C}$ and be sufficiently volatile at room temperature, $10^{-9}$ atm or more. With these conditions the only possible compounds that may be responsible for some of the mass lines in Table $\mathrm{I}$ are $\mathrm{Ru}(\mathrm{CO})_{5}$, $\mathrm{C}_{2} \mathrm{Cl}_{6}$ and $\mathrm{OsO}_{4}$. Setting aside the improbability of having chlorine, ruthenium, or osmium as impurities in the sample, the only masses from Table I that might be ascribable to ordinary compounds are 237, 241, and 254. The remaining 23 masses of Table I must correspond to species that formed and are stable at $1020-1050^{\circ} \mathrm{C}$ and that are volatile at room temperature, or represent random fortuitous signals. 
To distinguish between physically significant and random signals we apply the Poisson distribution function (11) to our data. We divide the entire range of masses investigated, 200 to 400 AMU, into 20 subdivisions, or bins, and we count the number of significant masses that appear in each bin (see Table III). If we assume that the probability of a random signal is independent of the mass value, then on the basis of pure chance, the average population of any one bin would be 1.15 mass values. From the Poisson distribution function it follows that the probability of finding by chance 8 or more mass entries in any one of the 20 bins is $6 \times 10^{-4}$. This strongly suggests that the mass values in the 231-240 bin are physically real. The other mass values in Table I, particularly those not in parentheses, may also be real but our data are too sparse to make any claim at this stage.

Fisher's theory holds that polyneutrons that have grown to large atomic masses can decay by $\beta$-emission and by fission until the number of protons and the number of neutrons correspond to the bands of stability shown in Figure 1. In which band a polyneutron ends its decay and exactly which atomic weight it acquires depend on the particular chain of $\beta$-decay and fission that it happens to follow, and thus is a stochastic process. Hence, there is no theoretical necessity that every experiment should give rise to the same mass distribution, although the masses that appear must lie within the bands of stability (Figure 1).

In the present stage of development of the theory, the decay ending with six protons, therefore carbon, can have various numbers of neutrons such that the atomic mass of neutronrich carbon can be 187 to $208 \mathrm{AMU}$. If correct, this means that heavy $\mathrm{CO}_{2}$ can range between 219 and 240 AMU. It is interesting and provocative that the mass bracket 231-240 lies within the range that Fisher's theory predicts for $\mathrm{CO}_{2}$ the carbon of which is a stable neutron-rich nuclide. It cannot be claimed that our work has proven the existence of neutron-rich nuclides. It is believed, however, that this work provides ample reason to take seriously the polyneutron theory of cold nuclear reactions and to attempt to devise an experimental technique to provide positive evidence for neutron-rich nuclides. This will be very difficult because of complete ignorance concerning the electronic transitions of the putative nuclides. Although difficult the search if successful would be very rewarding because a new branch of nuclear physics will thereby be established.

\section{ACKNOWLEDGMENTS}

The help of E. Larka, of the Department of Chemistry, with the mass spectroscopic analysis has been critical in this work and is gratefully acknowledged. The kindness of Professor A. Takahashi in supplying samples is much appreciated. I am grateful also for critical comments and advice from Dr. J.C Fisher. 
Table I.

Significant Masses Found in Room Temperature Gas Resulting From Oxidation at 1020-1050

\begin{tabular}{|c|c|c|c|}
\hline Pd, 0-113 & $\mathrm{T}-7$ & T-4 & $\mathrm{Ni}$ \\
\hline & (212) & & \\
\hline & (216) & & \\
\hline & & $222(\mathrm{~S})$ & \\
\hline & & $(233)(\mathrm{S})$ & \\
\hline & & 234 & \\
\hline & & $(235)(\mathrm{SS})$ & \\
\hline & $(236)(\mathrm{mS})$ & & \\
\hline \multirow[t]{10}{*}{238} & 237 & $238(\mathrm{SS})$ & 238 \\
\hline & 240 & & \\
\hline & (241) & & \\
\hline & & 247 & \\
\hline & & (252) & \\
\hline & & (254) & \\
\hline & $272(S)$ & & \\
\hline & & (275) & \\
\hline & & $277(\mathrm{SS})$ & \\
\hline & 292 & & \\
\hline \multirow[t]{5}{*}{299} & & & 315 \\
\hline & & & 316.5 \\
\hline & & & 330 \\
\hline & & $351(\mathrm{~S})$ & \\
\hline & & & $(360)$ \\
\hline
\end{tabular}

Notes: A conservative estimate of accuracy of these masses is \pm 0.2 AMU.

Mass values in parentheses are those for which the mass signal for the cathode sample was much stronger than the very weak signal in the corresponding blank (see Figure 3). Mass values without parentheses are those that do not show any signal at all in the corresponding blank (see Figure 3).

$\mathrm{S}$ signifies a strong signal, $\mathrm{SS}$ very strong, $\mathrm{mS}$ medium strong. The others represent weak signals but still significantly above noise. 
Table II.

Literature Mass Values of Compounds Whose Masses Are Within 0.9 AMU of the Significant Masses Found

\begin{tabular}{|c|c|c|}
\hline & \multicolumn{2}{|c|}{ Literature Value } \\
\hline Significant Mass Found & Mass & Compound \\
\hline 212 & 212.9 & $\mathrm{TaO}_{2}$ \\
\hline \multirow[t]{2}{*}{216} & 216.6 & $\mathrm{HgO}$ \\
\hline & 215.0 & $\mathrm{~S}_{2} \mathrm{O}_{5} \mathrm{Cl}_{2}$ \\
\hline \multirow[t]{2}{*}{234} & 234.4 & $\mathrm{NiFe}_{2} \mathrm{O}_{4}$ \\
\hline & 234.6 & $\mathrm{CoFe}_{2} \mathrm{O}_{4}$ \\
\hline \multirow[t]{2}{*}{235} & 234.8 & AgI \\
\hline & 234.7 & $\mathrm{NbCl}_{4}$ \\
\hline \multirow[t]{2}{*}{236} & 236.1 & $\mathrm{~N}_{2} \mathrm{O}_{3}\left(\mathrm{SO}_{3}\right)_{2}$ \\
\hline & 236.0 & $\mathrm{HgCl}$ \\
\hline 237 & 236.7 & $\mathrm{C}_{2} \mathrm{Cl}_{6}$ \\
\hline \multirow[t]{4}{*}{238} & 237.9 & $\mathrm{Se}_{2} \mathrm{O}_{5}$ \\
\hline & 237.7 & $\mathrm{FeCO}_{2} \mathrm{O}_{4}$ \\
\hline & 238.2 & $\mathrm{OsO}_{3}$ \\
\hline & 237.8 & $\mathrm{MoCl}_{4}$ \\
\hline \multirow[t]{3}{*}{240} & 239.5 & $\mathrm{~S}_{7} \mathrm{NH}$ \\
\hline & 240.3 & $\mathrm{Ni}_{3} \mathrm{~S}_{2}$ \\
\hline & 239.2 & $\mathrm{CuFe}_{2} \mathrm{O}_{4}$ \\
\hline \multirow[t]{6}{*}{241} & 241.6 & $\mathrm{TeF}_{6}$ \\
\hline & 241.7 & InI \\
\hline & 241.1 & $\mathrm{ZnFeO}_{4}$ \\
\hline & 241.8 & $\mathrm{Rb}_{2} \mathrm{Cl}_{2}$ \\
\hline & 241.1 & $\mathrm{Ru}(\mathrm{CO})_{5}$ \\
\hline & 240.8 & $\mathrm{Co}_{3} \mathrm{O}_{2}$ \\
\hline \multirow[t]{3}{*}{247} & 246.7 & $\left(\mathrm{NH}_{4}\right) \mathrm{H}_{5} \mathrm{TeO}_{6}$ \\
\hline & 247.8 & $\mathrm{Ag}_{2} \mathrm{~S}$ \\
\hline & 247.9 & $\mathrm{WS}_{2}$ \\
\hline 252 & 252.7 & $\mathrm{CHBr}_{3}$ \\
\hline \multirow[t]{3}{*}{254} & 254.2 & $\mathrm{OsO}_{4}$ \\
\hline & 254.3 & $\mathrm{OsS}_{2}$ \\
\hline & 253.8 & $\mathrm{MoOCl}_{4}$ \\
\hline 272 & 272.2 & $\mathrm{CdBr}_{2}$ \\
\hline
\end{tabular}


Table III.

Assignment of Found Significant Mass Values to Subdivisions by Decades of the Examined Mass Range

\begin{tabular}{|c|c|}
\hline Mass Bracket & $\begin{array}{c}\text { Number of } \\
\text { Significant Mass } \\
\text { Values }\end{array}$ \\
\hline $201-210$ & 0 \\
\hline $211-220$ & 2 \\
\hline $221-230$ & 1 \\
\hline $231-240$ & 8 \\
\hline $241-250$ & 1 \\
\hline $251-260$ & 1 \\
\hline $261-270$ & 0 \\
\hline $271-280$ & 3 \\
\hline $281-290$ & 0 \\
\hline 291-300 & 2 \\
\hline $301-310$ & 0 \\
\hline $311-320$ & 2 \\
\hline $321-330$ & 1 \\
\hline $331-340$ & 0 \\
\hline $341-350$ & 0 \\
\hline $351-360$ & 2 \\
\hline $361-370$ & 0 \\
\hline $371-380$ & 0 \\
\hline $381-390$ & 0 \\
\hline $391-400$ & 0 \\
\hline
\end{tabular}




\section{REFERENCES}

1. M. Fleischmann, S. Pons, J. Electroanal. Chem. 261, 301 (1989).

2. E. Storms, J. Sci. Explor. 10,\#2, 185 (1996).

3. J.C. Fisher, Fusion Technology 22, 511 (1992).

4. J.C. Fisher, submitted to Fusion Technology.

5. G.H. Miley and J.A. Patterson, Proc. 2nd Intnl. Conference on Low Energy Nuclear Reactions, Texas A\&M, Sept. 1996; in press, J. New Energy.

6. G.H. Miley, G. Narne, M.J. Williams, J.A. Patterson, J. Nix, D. Cravens, and H. Hora, Proc. 6th Intnl. Conference on Cold Fusion, Japan, October 1996, pp. 629-644.

7. T. Mizuno, T. Ohmori, and M. Enyo, J. New Energy 1, 23-27 (1996).

8. R.A. Oriani, J.C. Nelson, Sung-Kyu Lee, and J.H. Broadhurst, Fusion Technology 18, 652 (1990).

9. K. Yasuda, Y. Nitta, and A. Takahashi, ref. 6, pp. 36-43.

10. J.A. Patterson, "System for Electrolysis," U.S. Patent \#5, 494, 559, February 27, 1996.

11. C.G. Wohl, et. al., Revs. Mod. Phys. 56, No. 2, Part 2, S46 (1984). 


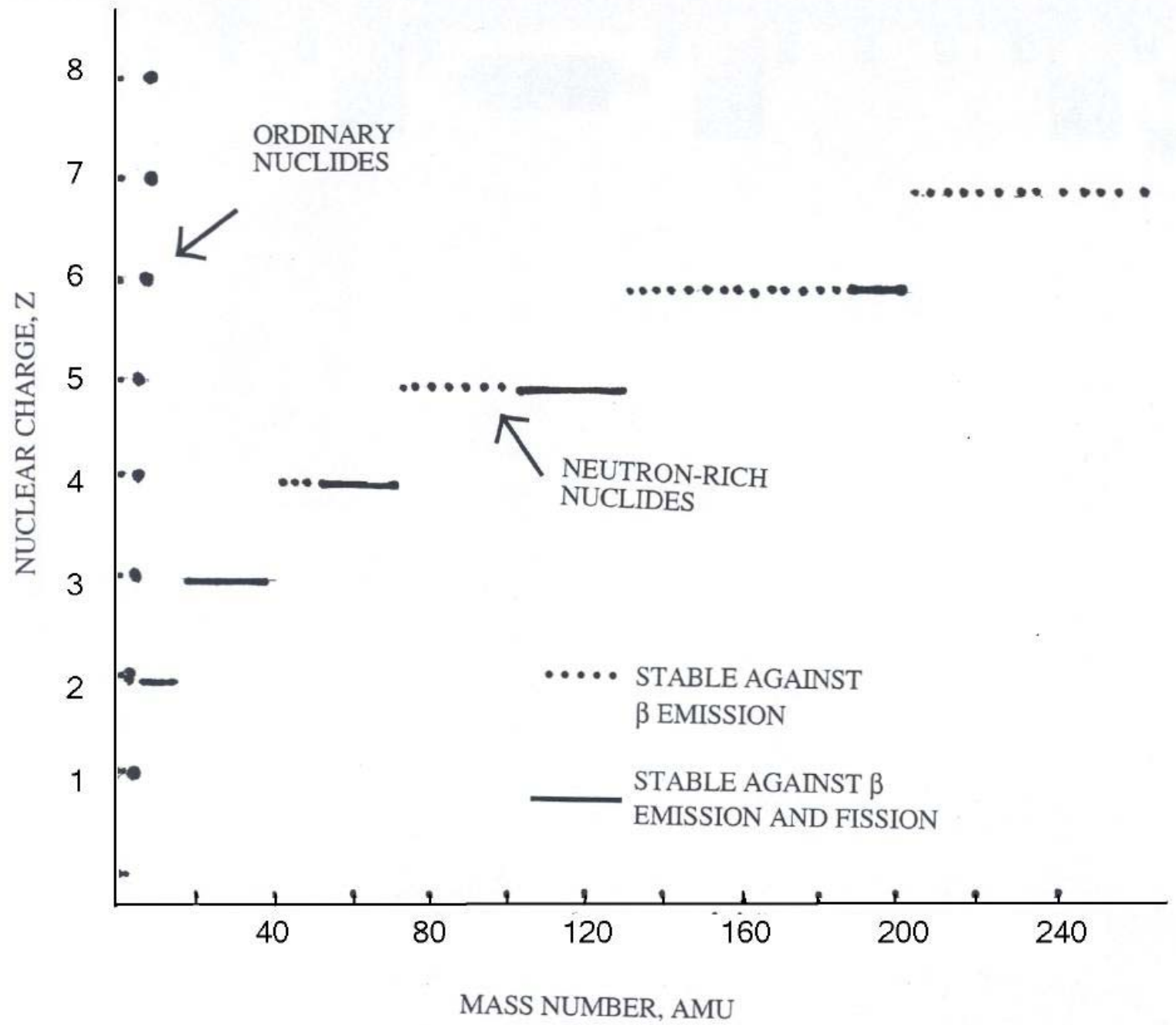

1. Nuclear charge vs. atomic mass ("valley of stability") of the ordinary nuclides and of the neutron-rich nuclides according to the theory of Fisher $(3,4)$. (After ref. 4). 


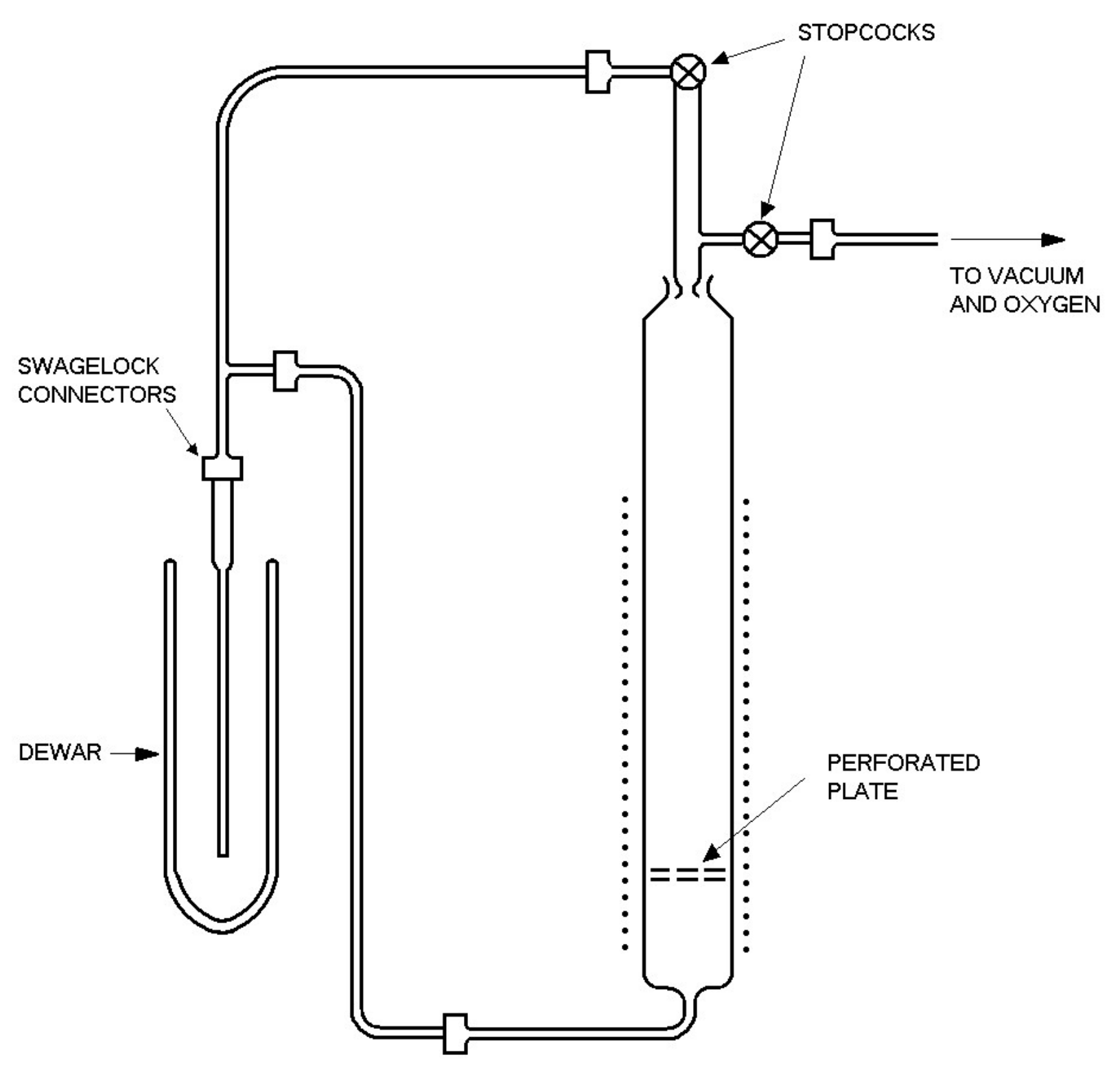

2. Schematic diagram of the apparatus employed for extracting carbon and nitrogen from cathodes previously used in electrolysis. 


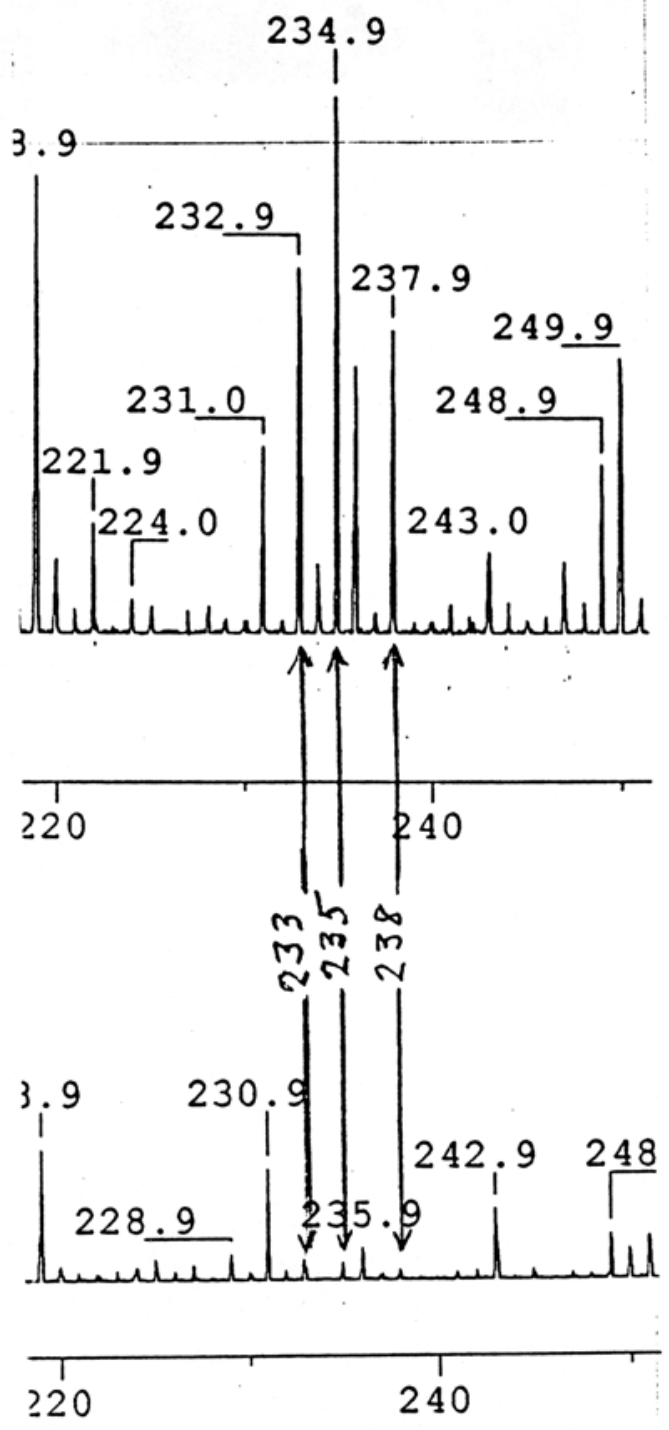

3. A segment of the mass spectrum from specimen T4 (the upper figure) juxtaposed with the corresponding segment of the blank spectrum. The line labeled 238 AMU is an example where the spectrum of the blank shows only noise at a mass value at which the specimen exhibits a strong signal. The lines labeled 233 and 235 are only slightly above noise in the spectrum of the blank, but are quite strong in the spectrum of the specimen. 